\title{
El Poder Político y Económico de Artefactos Líticos de Obsidiana del valle de Copán y la región de La Entrada, Honduras
}

Kazuo Aoyama

\section{Resumen}

El presente estudio proporciona un poco de luz sobre la discusión acerca de la naturaleza del antiguo urbanismo Mesoamericano. Los resultados del estudio sugieren que la administración de la obtención e intercambio de los núcleos pretrabajados de navajas de obsidiana de Ixtepeque, acompañada con otros factores, jugó un papel significativo en el desarrollo y mantenimiento del estado Clásico de Copán. La corte real institucionalizó los sistemas de la obtención y distribución intra e interregional de por lo menos una mercancía utilitaria como parte de la economía política o pública. El intercambio a larga distancia de pequeñas cantidades de mercancías de la élite tales como artefactos terminados de obsidiana verde, fue principalmente de importancia social y simbólica más que económica durante el período Clásico Temprano. Las élites emergentes en el valle de Copán participaron en redes de intercambio a larga distancia con el objeto de legitimar su autoridad y poder político. Sin embargo, el intercambio a nivel local fue mucho más crítico para el desarrollo del estado que el intercambio a larga distancia.

Palabras Clave: Copán, Obsidiana, intercambio, poder centralizado, economía política.

\section{Abstract}

This study sheds some light on the discussion about the nature of ancient Mesoamerican urbanism. The results of this research suggests that the management of the procurement and exchange of pre-worked obsidian polyhedral cores from Ixtepeque, accompanied by other factors, played a significant role in the development and maintenance of the Classic state of Copan. The royal court institutionalized the intra- and inter- procurement and distribution systems for at 
least one utilitarian commodity as part of the political or public economy. The longdistance exchange of small amounts of elite commodities such as finished artifacts made of green obsidian was mostly of social and symbolic importance rather than economic during the Early Classic period. The emergent elites of the Copan valley participated in long-distance exchange networks with the objective of legitimizing their authority and political power. However, local level exchange was more critical for the development of the state than the long- distance one.

Keywords: Copan, Obsidian, exchange, centralized power, political economy.

Kazuo Aoyama, Universidad de Ibaraki, Japón (Ibaraki University, Japan). 


\section{INTRODUCCIÓN}

Muchos estudiosos Mayistas han investigado sobre la arquitectura monumental, textos jeroglíficos, esculturas de piedra y cerámica. No obstante, el estudio de la lítica Maya no ha llamado mucha atención. Los artefactos líticos fueron los instrumentos principales entre los antiguos Mayas para construir esa gran civilización y no son perecederos. Lo importante es que podemos reconstuir algunos aspectos de la organización socioeconómica y urbanismo de la civilización Maya a través del estudio de la lítica. En el presente artículo, voy a presentar los resultados del estudio de la lítica Maya en el Valle de Copán y la región de La Entrada, Honduras.

El desarrollo de intercambio extenso frecuentemente juega un papel importante en la evolución de sociedades complejas, aunque la naturaleza exacta de dicho papel es ampliamente debatida. En el caso de la arqueología Maya, varios arqueólogos han usado artefactos de obsidiana para comprender mejor la naturaleza y el grado de los intercambios a larga distancia debido a las ventajas que ofrecen los análisis geoquímicos para determinar la procedencia de la obsidiana arqueológica (p.e., Braswell et al. 1994; McKillop 1989; Moholy-Nagy et al. 1984). Los arqueólogos Mayistas han utilizado gran parte de sus esfuerzos en estudios sobre intercambio a larga distancia; los intercambio intraregionales o locales han recibido mucha menos atención (Marcus 1983:477-480). Esto es parcialmente debido a que el enfoque primordial de las investigaciones en la arqueología Maya es todavía hacia sitios individuales, no regiones.

Existen marcadas diferencias de opinión con respecto a la naturaleza de la organización política Maya Clásica. Básicamente, un grupo de estudiosos reconstruye estados unitarios- 0 regionales con organización fuertemente centralizada y reconoce las mayores ciudades Mayas Clásicas como centros urbanos con economías administradas por solidaridad orgánica (Adams y Jones 1981; Chase y Chase 1996; Culbert 1991; Folan 1992). Otro grupo considera estados segmentados débilmente centralizados y propone que los estados Clásicos de las tierras bajas Mayas tenían funciones económicas débiles y que su poder fue fuertemente basado en la ideología (Ball y Taschek 1991; Demarest 1992; Fox y Cook 1996; Houston 1993). Algunos de los que proponen estos modelos están en gran desacuerdo sobre si los estados Clásicos Mayas tenían fuertes funciones administrativas de los sistemas de intercambio en bienes utilitarios o si las referidas funciones estaban débilmente desarrolladas. El presente estudio plantea las siguientes preguntas: (1) ¿Cuál fue la naturaleza de los sistemas de intercambio de 
obsidiana a través del tiempo en el valle de Copán y sus áreas vecinas? (2) ¿Cuál fue la relación entre los sistemas de intercambio de obsidiana y el desarrollo de la complejidad sociopolítica?

Para contestar las referidas preguntas usamos datos de 91,916 piezas de artefactos de lítica menor (piedra tallada) dentro y en los alrededores de Copán, ciudad localizada cerca de una de las principales fuentes de obsidiana para las tierras bajas Mayas ubicada en Ixtepeque, Guatemala. Los artefactos líticos fueron recolectados en diversas excavaciones realizadas en el núcleo urbano y la zona rural del valle de Copán y otros centros menores en las regiones vecinas. Se enfatizó en los patrones de distribución de artefactos de obsidiana con el fin de entender mejor la naturaleza y papel del intercambio regional e inter-regional así como el intercambio a larga distancia en el desarrollo de un estado de Copán. Estos artefactos, relacionados con los períodos Preclásico Temprano hasta el Postclásico Temprano (1400 A.C. 1100 D.C.), pueden servir como indicadores sensibles para la reconstrucción de un aspecto de los patrones cambiantes a largo plazo de los sistemas de intercambio Maya precolombino en y alrededor de Copán. También se estudió el urbanismo Clásico Maya mediante el examen del rol que juegan los centros en los sistemas de asentamiento regional, además de las interacciones socioeconómicas entre el centro y los sitios menores que los rodeaban.

Los antiguos habitantes del valle de Copán importaron obsidiana de al menos siete fuentes geológicas: Ixtepeque, El Chayal, y San Martín Jilotepeque en Guatemala; La Esperanza en Honduras; Pachuca y Zaragoza en México Central y Ucareo, Michoacan. Entre ellas, cerca del $100 \%$ de la obsidiana vino de la fuente más cercana, Ixtepeque, durante toda la secuencia precolombina. La distancia en línea recta desde el valle de Copán hasta Ixtepeque $(80 \mathrm{~km})$ es considerablemente más corta que la mayoría de las áreas de las tierras bajas Mayas. Debido a su cercanía, los antiguos habitantes del valle de Copán pudieron haber tenido acceso directo a la referida fuente de obsidiana. También, los nódulos de sílex de hasta $50 \mathrm{~cm}$ de diámetro se encuentran disponibles en abundancia en el valle de Copán. Las unidades domésticas individuales colectaron sílex principalmente para manufacturar lascas a percusión no especializadas.

La región de La Entrada está localizada a $50 \mathrm{~km}$ al noreste del valle de Copán, constituyendo parte de la periferia suroriental de las tierras bajas Mayas. Nuestro programa de recorrido regional localizó un total de 635 sitios precolombinos en un área de 150 km² (Inomata y Aoyama 1996; Nakamura et al. 1991). Las unidades 
políticas de la región de La Entrada tuvieron su apogeo durante el período Clásico Tardío e interactuaron activamente con el estado de Copán. Dicha región fue gobernada no por un poder político unido sino por varias unidades políticas de poder similar.

\section{METODOLOGÍA}

Clasificamos todos los artefactos de lítica menor mediante tipologías tecnológicas (figura 1). Se reconstruyeron los sistemas de intercambio de obsidiana mediante la combinación de análisis tecnológico y determinación de fuentes. Identificamos las fuentes de artefactos de obsidiana mediante la combinación de análisis de activación de neutrones y examen visual (Aoyama 1994, 1996, 1999, 2001a). Se llevaron a cabo exámenes visuales de todos los artefactos de obsidiana. Hicimos esto comparando artefactos de obsidiana con muestras de referencia que exhiben toda la gama de variabilidad óptica de fuentes de obsidiana precolombinas en México, Guatemala, y Honduras. La precisión de mi análisis visual fue confirmada mediante una prueba a ciegas de 100 artefactos de obsidiana de la región de La Entrada utilizando el análisis de activación de neutrones, el cual fue realizado por Michael D. Glascock de la Universidad de Missouri. Los resultados de la prueba a ciegas indicaron una tasa de precisión de $98 \%$. Más importantemente, investigadores independientes han demostrado que, por lo menos para algunas colecciones de artefactos de obsidiana Maya, el análisis visual es tanto reproducible como preciso (Braswell et al. 2000). 


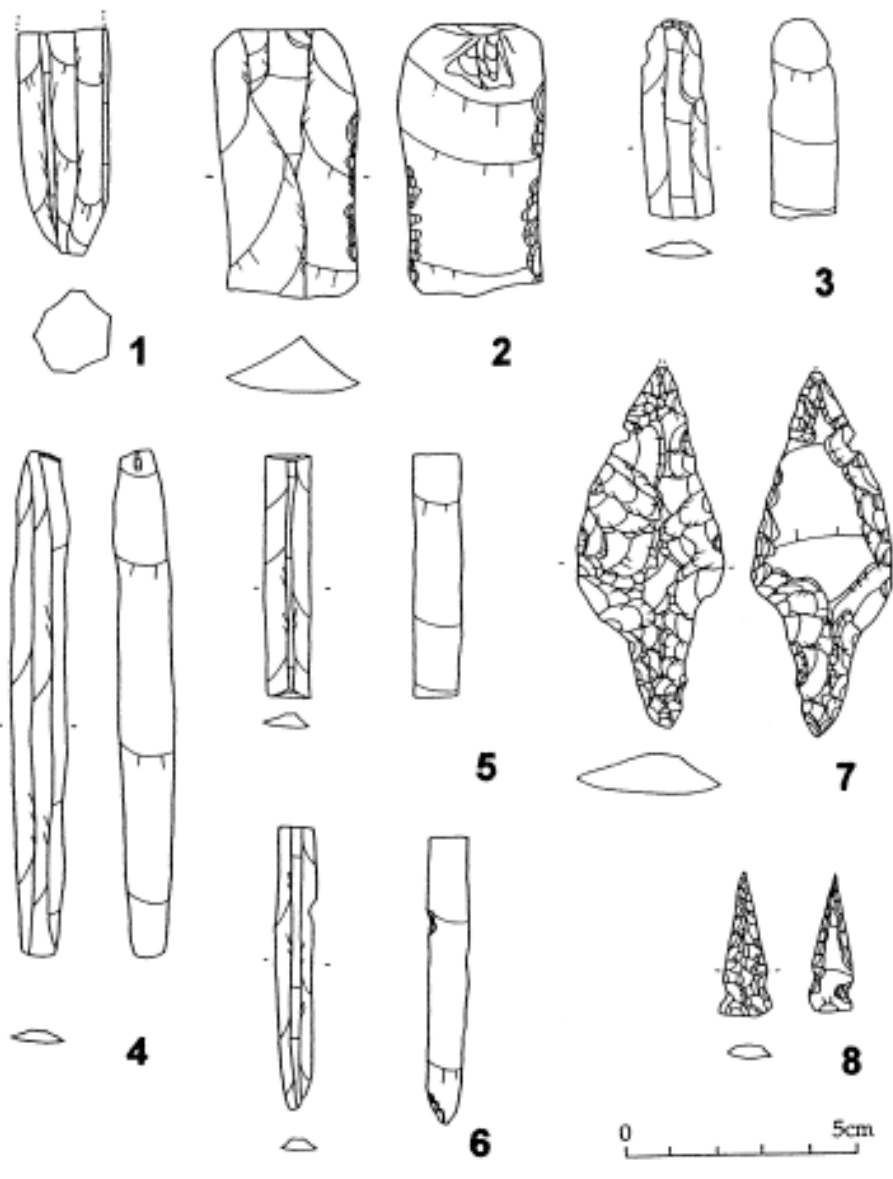

Figura 1. Artefactos de Obsidiana del Clásico del valle de Copán. (1) fragmento de núcleo poliédrico; (2) macronavaja; (3-6) navajas prismáticas; (7) punta bifacial;

(8) punta de navaja prismática.

En 1987 se condujo un estudio experimental intensivo de microhuellas de uso sobre obsidiana y sílex con el fin de establecer un marco de referencia para interpretar el uso de las herramientas de piedra entre los antiguos Mayas (Aoyama 1989). Los resultados de 267 experimentos de replicación llevados a cabo con una gama de materiales de trabajo permitieron la identificación de los patrones de microhuellas de uso basados en el método de gran alcance. Entre 1987 y 1995 analizamos microhuellas de uso sobre un total de 3,232 artefactos de lítica menor del Valle 
de Copán y Región de La Entrada, Honduras (Aoyama 1995, 1999, 2001b). El instrumento usado en el presente estudio fue un microscopio metalúrgico de 50-500 de aumento con un accesorio de luz incidente (OLYMPUS BX60M). La magnificación de 200x fue la más frecuentemente usada. Los patrones de microhuellas de uso fueron documentados con un sistema fotomicrográfico Olympus PM-10M conectada a una cámara (OLYMPUS C-35DA-2).

\section{Período Preclásico (1400 A.C. - 50 D.C.)}

Los artefactos de obsidiana del Preclásico Temprano (1400-900 A.C.) constituyen hasta la fecha uno de los artefactos de obsidiana más tempranos provenientes de depósitos estratificados en las tierras bajas Mayas. Debido al alto porcentaje de artefactos de obsidiana de Ixtepeque con corteza (27\%), parece que la obsidiana de Ixtepeque se importó en forma de grandes lascas o pequeños nódulos. No hay evidencias sobre tecnologías de núcleo-navajas o bifacial. Más bien parece que lascas no especializadas fueron producidas mediante una combinación de percusión directa y técnica bipolar.

Durante el período Preclásico Medio (900-300 A.C.) pequeñas cantidades de navajas prismáticas de obsidiana de Ixtepeque fueron importadas como productos terminados. Todavía no tenemos evidencias de la producción local de navajas prismáticas. A pesar de todo, el intercambio de navajas prismáticas fue una empresa poco voluminosa y no sobrepasó la industria preexistente de lascas no especializadas. Además, la industria de lascas no especializadas de sílex no cambió nada en respuesta a la introducción de navajas prismáticas de obsidiana. Con base al bajo porcentaje general de navajas prismáticas en los artefactos de obsidiana (2.7\%) y alto porcentaje de artefactos de obsidiana de Ixtepeque con corteza $(20.5 \%)$, parece que la obsidiana de Ixtepeque continuó siendo importada principalmente como grandes lascas o pequeños nódulos.

Aún durante el período Preclásico Tardío (300 A.C. - 50 D.C.), debido al bajo porcentaje general de navajas prismáticas en los artefactos de obsidiana (8.8\%) además del alto porcentaje de artefactos de obsidiana de Ixtepeque con corteza (19.5\%), parece que la obsidiana de Ixtepeque fue importada principalmente en forma de grandes lascas o pequeños nódulos como se hizo en los períodos anteriores. Además, pequeñas cantidades de navajas prismáticas y macronavajas parecen haber sido importadas como artefactos terminados. La falta de evidencia de la importación de macronúcleos de obsidiana y producción local de navajas 
prismáticas está asociada con la reducción de la población y la organización sociopoítica de nivel simple en el valle de Copán durante este período. La situación de Copán es una anormalidad en el área Maya y sus alrededores debido a que la tecnología de navajas ya estaba siendo utilizada en las sociedades complejas contemporáneas, por ejemplo, en Chalchuapa (Sheets 1978) y en Quelepa, El Salvador (Braswell et al. 1994).

\section{Período Protoclásico (50 - 400 d.C.)}

Al comienzo del Protoclásico Temprano (50 - 150 D.C.), Copán parece haber mantenido una organización sociopolítica de nivel simple. Los habitantes locales en ese momento todavía importaron la obsidiana de Ixtepeque principalmente en forma de grandes lascas o pequeños nódulos además de una pequeña cantidad de navajas prismáticas terminadas (Braswell et al. 1996).

Las inscripciones en la Estela I en Copán citan un posible gobernante y un evento el cual tomó lugar en 159 D.C. que puede estar relacionado con la fundación de Copán como un reino (Stuart 1992:171). Durante el período Protoclásico Tardío (150-400 D.C.) los datos arqueológicos claramente indican que la población del valle creció substancialmente. Con base a la arquitectura diferencial y las ofrendas funerarias, Fash (1991:74) sugiere al menos dos niveles socioeconómicos en el valle de Copán. La presencia de núcleos poliédricos y grandes desechos de talla relacionados con la tecnología núcleo-navaja sugiere el comienzo de la importación de núcleos pretrabajados de navajas al valle de Copán durante ésta fase. Otras líneas de evidencia para esto incluyen un dramático aumento en el porcentaje de navajas prismáticas en los artefactos de obsidiana (37.7\%) y una disminución en el porcentaje de la obsidiana de Ixtepeque con corteza (11.8\%), en comparación al Preclásico Tardío.

Aunque la muestra de obsidiana del Protoclásico Tardío es pequeña, el grado relativo de disponibilidad de navajas fue asociado con el cambio en el patrón de asentamiento. El posible control político sobre la distribución de navajas se puede inferir con la distribución desigual de dichos artefactos en Copán. Tanto los residentes del Grupo Principal como los de las estructuras estucadas de bloques de piedra canteada en el Grupo 10L-18 tuvieron mayor acceso a las navajas prismáticas de obsidiana de Ixtepeque que las otras unidades domésticas fuera del Grupo Principal. Durante el período Protoclásico Tardío uno de los gobernantes de Copán pudo haber comenzado a administrar la obtención de núcleos pretrabajados de navajas de obsidiana de Ixtepeque y la producción local de navajas prismáticas 
en favor de su comunidad como un medio para consolidar y legitimar su propia autoridad política. La decisión no parece haber sido motivada por un deseo de economizar u obtener una ganancia. Las navajas prismáticas podrían haber sido distribuidas como un tipo de regalo político para atraer seguidores y aliados. Si fuera el caso, la adopción de la tecnología de navajas pudo haber empezado como el resultado, más que la causa, del desarrollo sociopolítico en el Valle de Copán.

\section{Período Clásico Temprano (400 - 600 D.C.)}

K'inich Yax K'uk' Mo' fundó una nueva dinastía real en Copán en el 426 D.C. (Fash 1991). Un incremento significativo en el porcentaje de navajas prismáticas en artefactos de obsidiana (64.8\%) así como la correspondiente disminución de obsidiana de Ixtepeque con corteza (2.7\%) durante el período Clásico Temprano pueden señalar un marcado incremento en la importación de núcleos pretrabajados de navajas y la producción local de navajas prismáticas. Estos cambios pudieron haber sido el resultado del desarrollo sociopolítico que culminó en la formación del estado de Copán al final del siglo siete D.C. (Fash 1991:112), como podemos ver otros cambios mayores en patrones de asentamiento, arquitectura monumental, textos jeroglíficos, artes, cerámica y tradiciones funerales. No hay evidencia de la producción de lítica menor a tiempo completo u otra producción no dirigida a la subsistencia con artefactos líticos en la antigua ciudad de Copán aún durante el período Clásico. Las extensas excavaciones en el Grupo Principal de Copán han localizado dos residuarios de talleres de producción de navajas prismáticas del período Clásico Temprano (Aoyama 2005a). Debido a que Copán fue abandonado mucho más gradualmente que Aguateca (Aoyama 2007), el papel de la producción artesanal en el desarrollo sociopolítico pudo haber sido considerablemente menospreciado.

Mientras que casi toda la obsidiana fue obtenida de Ixtepeque, como en los períodos anteriores, al comienzo del Período Clásico Temprano K'inich Yax K'uk' Mo' pudo haber comenzado a obtener pequeñas cantidades de artefactos terminados hechos de obsidiana verde de Pachuca del México Central (principalmente navajas prismáticas, pero también pequeñas cantidades de puntas bifaciales). La importación de los artefactos de obsidiana verde no fue substancial. No obstante, el porcentaje de obsidiana verde en artefactos de obsidiana (9.8\%) de la Estructura Yax, que fue construida bajo la supervisión de K'inich Yax K'uk' Mo', es uno de los más altos en las tierras bajas Mayas durante el período Clásico. 
Una posible interpretación del alto porcentaje de obsidiana verde en la Estructura Yax es que Yax K'uk' Mo' era de Teotihuacan o de un centro como Tikal o Kaminaljuyú los cuales tenían una fuerte, y posible interacción directa con Teotihuacán. Es también posible que él fue un Copaneco local quien usó los artefactos de obsidiana verde relacionados con Teotihuacán para legitimar su autoridad y poder político. Sin embargo, evidencias arqueológicas y epigráficas sustentan la hipótesis que K'inich Yax K'uk' Mo' fue un extranjero que llegó a Copán (Sharer et al. 1999:20; Stuart 2000:492).

La distribución espacial restringida de la obsidiana verde en el valle de Copán durante el período Clásico Temprano sugiere que los artefactos de obsidiana verde pueden haber sido mercancías de la élite. La dinastía de K'inich Yax K'uk' Mo' pudo haberlos redistribuido a los líderes locales como dones de la élite para asegurar y solidificar la alianza con los seguidores y aliados. Los artefactos de obsidiana verde están concentrados en el Grupo Principal y las cercanías inmediatas del núcleo urbano mientras ellos estaban virtualmente ausentes en las áreas rurales del valle de Copán. Es significante destacar que vasijas policromadas importadas de otras partes del área Maya también tenían una distribución limitada en el valle de Copán. Las referidas vasijas eran raras incluso en el núcleo urbano, pero ellas eran casi ausentes fuera de esta área (Bill 1997:543). Dicha distribución espacial se sobrepone a la de los artefactos terminados de obsidiana verde y refuerza la hipótesis de que los artefactos de obsidiana verde fueron mercancías de la élite. Los análisis de microhuellas y análisis contextuales en artefactos de obsidiana verde sugieren que estos artefactos fueron principalmente "mercancías utilitarias de la élite," i.e., mercancías utilitarias consumidas por las élites en el valle de Copán durante el período Clásico Temprano. Los resultados del análisis de microhuellas sobre las navajas prismáticas de obsidiana verde indican que ellas fueron usadas para diversas tareas mundanas, es decir, cortar carne o cuero, raspar cuero, cortar, aserrar o grabar madera u otras plantas (Aoyama 1999:107). La gran mayoría de los artefactos de obsidiana verde vienen de basureros domésticos y rellenos de construcción; las frecuencias son mucho más bajas en escondites y entierros.

El uso o exhibición de estas pequeñas cantidades de bienes procedentes de intercambios a larga distancia tuvo significado social y simbólico, más que económico (Drennan 1991:281). El color mismo de la obsidiana verde pudo haber tenido importancia social y simbólica (Sharer 1983:255). El control de los materiales exóticos y conocimientos esotéricos de lugares sagrados distantes tal como Teotihuacán pueden haber sido cruciales para legitimar la autoridad y poder político de los gobernantes emergentes en el Valle de Copán durante el período Clásico 
Temprano. Sin embargo, debido a que el porcentaje de obsidiana verde en todos los artefactos de obsidiana disminuyó a través de la secuencia arquitectónica en la Acrópolis del Grupo Principal de Copán, el papel de la obsidiana verde parece haber decrecido a través del tiempo. Dicho patrón de disminución de uso pudo haber sido parcialmente el resultado de un cambio de conexiones de la élite. Si K'inich Yax K'uk' Mo' hubiese sido un extranjero, este patrón puede haber resultado de la asimilación de la dinastía de K'inich Yax K'uk' Mo' a la cultura local a través del tiempo. A pesar de todo, gobernantes más tardíos puede que hayan requerido menos símbolos externos de refuerzo de poder, a medida que la organización sociopolítica local se fue consolidando. Para el período Clásico Tardío, los gobernantes con bases consolidadas de poder local cesaron de importar obsidiana verde.

\section{Período Clásico Tardío (600 - 900 D.C.)}

La obsidiana de Ixtepeque fue importada al valle de Copán durante el período Clásico Tardío principalmente en forma de núcleos pretrabajados de navajas, tal como se hizo en el período anterior. Los análisis de microhuellas y contextos indican que las navajas prismáticas de obsidiana de Ixtepeque no fueron mercancías de lujo sino fundamentalmente mercancías utilitarias. Tanto las élites como los comunes las usaron para una amplia gama de trabajos diarios: cortar, aserrar, tallar o grabar madera u otras plantas; cortar y raspar carne o cuero; cortar, aserrar y tallar concha, hueso 0 asta y un mucho menor grado para rituales de sacrificio (Aoyama 1999:133). Aparentemente, todas las unidades domésticas tuvieron acceso a navajas prismáticas terminadas hechas de obsidiana de Ixtepeque, tal como se hizo en los períodos anteriores. La gran mayoría de ellas aparecen en contextos domésticos, con una ocurrencia mucho menor en contextos ceremoniales.

No todas las unidades domésticas tuvieron acceso a los núcleos pretrabajados de navajas o pudieron producir navajas prismáticas. Algunos de los campesinos más pobres en las áreas rurales obtuvieron una pequeña cantidad de navajas prismáticas terminadas hechas de obsidiana de Ixtepeque, además de pequeños nódulos y grandes lascas para la producción no especializada de lascas a percusión. Sheets (1983:96-97) sugiere que mientras la élite Maya Clásica en Quiriguá importó macronúcleos de obsidiana para la producción especializada de navajas prismáticas, la gente que vivía en las áreas rurales obtuvo pequeños guijarros de obsidiana para la producción informal de lascas a percusión. De la misma manera, es posible diferenciar entre la industria de núcleo-navaja y la de lascas a percusión en el valle de Copán durante el período Clásico Tardío. 
Los datos sobre artefactos de lítica menor de basureros primarios en el valle de Copán del Clásico Tardío indican una distribución desigual, sugiriendo que los gobernantes tuvieron mayor acceso a la obsidiana de Ixtepeque que los otros habitantes del valle. La densidad de obsidiana en los basureros del Grupo Principal es considerablemente mayor que en los de las residencias grandes y pequeñas, indicando que la familia real desechó más obsidiana y presumiblemente mayor acceso que otras casas en el valle de Copán. Además, el porcentaje de obsidiana en todos los artefactos de lítica menor del Grupo Principal (Media $=94.9$, D.S. $=$ 4.2) es considerablemente mayor que el de las residencias grandes (Media $=85.4$, D.S. $=9.6$ ) y el de las residencias pequeñas (Media = 69.3, D.S. $=19.6$ ), mientras que el de sílex local aumenta del Grupo Principal a las residencias pequeñas. Esto podría indicar una mayor disponibilidad de obsidiana de acuerdo a la riqueza de las unidades domésticas. Otra línea de evidencia para el acceso preferencial de los gobernantes a la obsidiana de Ixtepeque es un escondite del Clásico Tardío consistente en 700 macronavajas y macrolascas extraordinariamente grandes (casi $30 \mathrm{~cm}$ de largo y $15 \mathrm{~cm}$ de ancho). Dicho escondite (figura 2), fue depositado durante el reinado del gobernante 12 o 13 en el centro de la Plaza Principal donde contenía numerosas estelas y otros monumentos de piedra y fue sin duda alguna un espacio para presentaciones teatrales. Cantidades tan grandes de macronavajas y macrolascas de gran tamaño no han sido descubiertas fuera del Grupo Principal en el valle de Copán o en cualquier otra parte de las tierras bajas Mayas. La presentación teatral y ritual de depósitos de obsidiana en la Plaza Principal tuvo que haber reforzado el poder político y económico del gobernante.

Los patrones diferenciales de distribución de obsidiana pudieron haber resultado parcialmente por el mayor poder de adquisición de las unidades domésticas involucradas. No obstante, creo que los referidos patrones podrían indicar un mecanismo centralizado de dispersión, es decir, la distribución de núcleos pretrabajados de navajas de obsidiana de Ixtepeque fue institucionalizada por la corte real como parte de la economía política. Esta interpretación claramente contradice un estudio previo conducido por Mallory (1984) en el cual sugirió que la producción de navajas prismáticas de obsidiana fue llevada a cabo en todas las unidades domésticas en Copán y que la obsidiana fue igualmente disponible para todas las clases sociales. Es de hacer notar que las muestras de obsidiana que Mallory estudió nunca fueron suficientes para un estudio a nivel regional y no incluyeron ningún artefacto del Grupo Principal. 

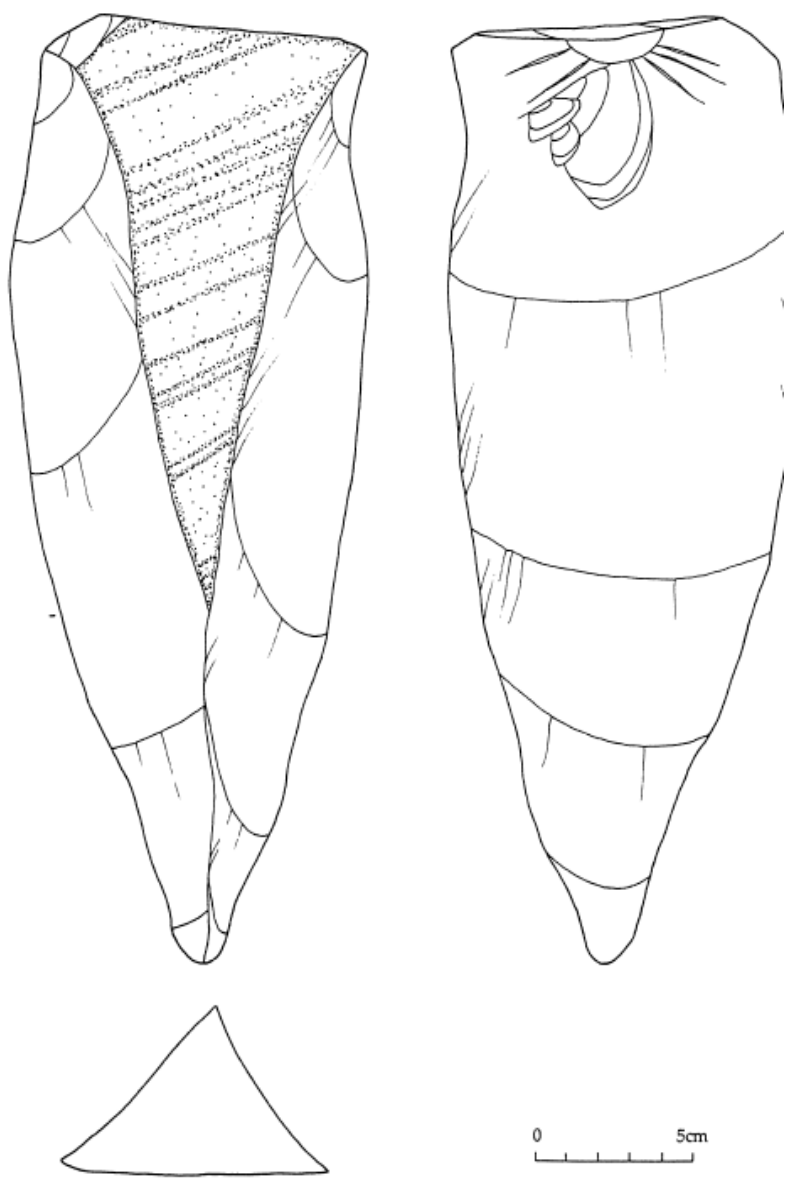

Figura 2. Una macronavaja muy grande de la Plaza Principal de Copán, Clásico Tardío.

Los resultados de mi estudio sugieren que la antigua ciudad de Copán funcionó como un centro de distribución de núcleos pretrabajados de obsidiana de Ixtepeque hacia los sitios más pequeños en el valle de Copán y otros centros menores en las regiones vecinas (Aoyama et al. 1999). Una fuerte caída en la distribución de la obsidiana de Ixtepeque está a aproximadamente $60 \mathrm{~km}$ del Grupo Principal de Copán. En otras palabras, la obsidiana de Ixtepeque dominó virtualmente toda la region de La Entrada excepto en el extreme norte de la región, en donde la industria de lascas a percusión de obsidiana de San Luis dominó la industria de núcleonavaja de obsidiana de Ixtepeque. La fuente de San Luis está localizada a $30 \mathrm{~km}$ de la región de La Entrada y pequeños nódulos de obsidiana de San Luis fueron 
usados exclusivamente para la producción no especializada de pequeñas lascas a percusión. Es posible que la fuerte disminución de la obsidiana de Ixtepeque en la región de La Entrada fuese el resultado de factores políticos. Esta distancia es paralela al radio hipotético de los estados regionales del Clásico Maya.

Los gobernantes locales de los centros de la parte sur de la región de La Entrada, tales como Los Higos y El Abra, parecen haber obtenido obsidiana principalmente en forma de núcleos pretrabajados a través del intercambio directo con el estado de Copán, distribuyéndolos a los sitios más pequeños de la región de La Entrada. El porcentaje de obsidiana de Ixtepeque, la fuente lejana y la obsidiana de alta calidad, aumenta en relación a la categoría de sitio de pequeño a grande, mientras que el de la obsidiana de San Luis, la fuente cercana y la obsidina de baja calidad, disminuye de los sitios grandes a los pequeños. Esto podría indicar una mayor disponibilidad de la obsidiana de Ixtepeque de acuerdo a la posición jerárquica de los habitantes de un sitio.

Las inscripciones en el vaso de alabastro de El Abra, el mayor centro en la región de La Entrada, proporcionan una línea explícita de evidencia con relación a la interacción económica directa entre un dirigente local y el gobernante 16 de Copán, Yax Pasaj Chan Yopaat. Según David Stuart (1995), luego de la primera oración, la cual registra la fecha 7 Ets'nab 11 Yax, o sea, 9 de Agosto de 775, una segunda oración se refiere a una danza ritual del gobernante 16 de Copán relacionada con el recibimiento de 17 cargas de "tributo." Dado que la cantidad de tierra disponible para la agricultura estaba siendo reducida por los asentamientos humanos en el valle de Copán, tal tributo puede haber incluido alimentos. La región de La Entrada está lo suficientemente cerca para hacerla un punto de origen de alimentos básicos importados al valle de Copán. Por lo tanto, el intercambio de obsidiana de Ixtepeque podría reflejar la movilización de sobreproducción de alimentos.

Con base a los datos de obsidiana de los basureros primarios de la Acrópolis de Copán, la disponibilidad general de obsidiana parece haber decrecido de forma marcada en la última mitad del período Clásico Tardío. Los últimos gobernantes tuvieron menos acceso a la obsidiana y pudieron haber tenido más dificultades en la administración de los sistemas de intercambio inter e intraregional que sus predecesores inmediatos en la cumbre política de Copán. Tal evidencia podría sugerir que el período Clásico Tardío fue un período de gran inestabilidad interna. Lo que implica esto es que el decaimiento de la autoridad política central podría no haber sido un acontecimiento tan súbito. Aún más importante, la producción de puntas de navaja prismática de obsidiana, así como puntas bifaciales de obsidiana 
y sílex, incrementó hacia el final del período Clásico Tardío (Aoyama 2005 b). Dicho incremento de ambos tipos de puntas puede ser tomado como evidencia para una escalada en la competencia intra- y/o intervalle.

\section{Período Postclásico Temprano (950 - 1100 D.C.)}

Luego de la desaparición de la autoridad dinástica centralizada en el siglo nueve, el sistema de obtención y distribución intraregional de núcleos pretrabajados de obsidiana de Ixtepeque se vino abajo, lo que resultó en el cese de la producción local de navajas prismáticas en el valle de Copán. Los habitantes del valle de Copán durante el período Postclásico Temprano regresaron al modo Preclásico de obtención y producción de obsidiana de Ixtepeque. Ellos obtuvieron esta obsidiana principalmente como pequeños nódulos y grandes lascas. Los Copanecos del Postclásico Temprano también recuperaron pedazos grandes de obsidiana de depósitos más tempranos en Copán y los usaron como núcleos simples para manufacturar pequeñas lascas no especializadas a percusión.

Los mencionados cambios están reflejados en una dramática disminución en el porcentaje de navajas prismáticas en los artefactos de obsidiana de Ixtepeque (del 69.4 al 23.4\%), además de un incremento significativo de la obsidiana de Ixtepeque con corteza (del 2.8\% al 9.7\%) del período Clásico Tardío al Postclásico Temprano. El decaimiento de la tecnología de navajas durante el período Postclásico Temprano refuerza el argumento de que la corte real institucionalizó la obtención y distribución de núcleos pretrabajados de navajas de obsidiana de Ixtepeque durante el período Clásico.

No obstante, los Copanecos del Postclásico Temprano no estaban aislados de otras regiones, sino que participaron en las crecientes redes de intercambio a larga distancia de la Mesoamérica Postclásica. Aunque Ixtepeque fue aún la fuente de obsidiana más comúnmente usada, ellos importaron pequeñas cantidades de navajas prismáticas terminadas de obsidiana de Pachuca y Ucareo de las tierras altas de México. La notoria presencia de obsidiana de las tierras altas de México en el gran centro de Chichén Itzá (Braswell y Glascock 1995) además de los sitios costeros más pequeños en las tierras bajas Mayas (McKillop 1989) durante el período Postclásico Temprano podría implicar que esta distribución se volvió más amplia y descentralizada. Los Copanecos del Postclásico Temprano también usaron cantidades limitadas de alfarerías importadas como Tohíl Plomizo, Anaranjado Fino y Policromado Las Vegas además de la cerámica utilitaria de la 
fase Ejar del Postclásico Temprano (Manahan 2004). Sin embargo, la escala del intercambio a larga distancia nunca fue grande. Los análisis de microhuellas y contextos de las navajas prismáticas de obsidiana de las tierras altas de México indican que ellas eran esencialmente mercancías utilitarias en el valle de Copán durante el período Postclásico Temprano. Sin embargo, ni siquiera el incremento del intercambio a la larga distancia alivió la carestía política y económica traída por el colapso de la autoridad dinástica centralizada en Copán.

\section{IMPLICACIONES PARALA ORGANIZACIÓN SOCIOECONÓMICAY URBANISMO DE UN ESTADO CLÁSICO MAYA}

Los resultados del presente estudio sugieren que la administración de la obtención e intercambio de los núcleos pretrabajados de navajas de obsidiana de Ixtepeque, acompañada con otros factores, jugó un papel significativo en el desarrollo y mantenimiento del estado Clásico de Copán. La corte real institucionalizó los sistemas de la obtención y distribución intra e interregional de por lo menos una mercancía utilitaria (i.e., núcleos pretrabajados de obsidiana de Ixtepeque) como parte de la economía política o pública. El intercambio a larga distancia de pequeñas cantidades de mercancías de la élite tales como artefactos terminados de obsidiana verde, fue principalmente de importancia social y simbólica más que económica durante el período Clásico Temprano. Las élites emergentes en el valle de Copán participaron en redes de intercambio a larga distancia con el objeto de legitimar principalmente su autoridad y poder político. Los artefactos de obsidiana verde del México Central pueden haber sido principalmente una mercancía utilitaria de la élite, y la dinastía de K'inich Yax K'uk' Mo' puede haberlos redistribuido como dones para atraer seguidores y aliados. Sin embargo, el intercambio a nivel local fue mucho más crítico para el desarrollo del estado que el intercambio a larga distancia.

El presente estudio sobre el consumo e intercambio de obsidiana en Copán proporciona un poco de luz sobre la discusión actual acerca de la naturaleza del antiguo urbanismo Mesoamericano. Sanders y Webster (1988) tienden a ver sólo los centros más grandes y más densamente poblados como "verdaderamente urbano," y argumentan que casi todas las antiguas ciudades Mesoamericanas (exceptuando unas pocas ciudades administrativas tales como Teotihuacán) corresponden al tipo real-ritual (figurando Copán como un ejemplo fundamental), en el cual las unidades políticas fueron organizadas con base a líneas de parentesco. 
La sociedad Clásica de Copán fue internamente heterogénea como lo fue la sociedad de Teotihuacán. Esto se desprende de la gran variabilidad en riqueza representada por las diferencias en el acceso a la obsidiana de Ixtepeque además de la dicotomía entre la industria urbana de núcleo-navaja y la rural de lascas a percusión. Mientras que Teotihuacán tuvo instituciones económicas más fuertes y desarrolladas, un gobierno más centralizado y una población mucho más grande que la de Copán, ambas ciudades se caracterizaron por actividades económicas diferentes. Así que las diferencias entre las economías administrativas de Teotihuacán y Copán fue una cuestión de escala en vez que de tipo.

Sin tomar en cuenta el tamaño de la población, las antiguas ciudades Mayas sirvieron una gama más amplia de funciones administrativas y económicas que la ciudad real-ritual. Los gobernantes de Copán aprovecharon su inusual ubicación cerca de recursos altamente localizados, es decir, la obsidiana de Ixtepeque. La obtención directa de obsidiana de alta calidad señala una diferencia significativa entre Copán y otras grandes ciudades Mayas. Mientras que los gobernantes de Copán fueron exportadores además de consumidores de núcleos de navajas de obsidiana, los gobernantes en la mayoría de las tierras bajas Mayas fueron principalmente consumidores de núcleos de navajas de obsidiana e intermediarios dentro de las redes de intercambio a larga distancia. En suma, el estado Clásico de Copán tuvo por lo menos una función administrativa en la obtención y distribución de núcleos pretrabajados de navajas de obsidiana de Ixtepeque, un elemento importante de conjuntos utilitarios del antiguo Copán. El estado de Copán obtuvo núcleos de navajas de obsidiana de Ixtepeque, los distribuyó a los nobles que vivían en el valle de Copán y los exportó a los gobernantes locales de centros más pequeños en las regiones vecinas. Con respecto a esto, el estado Clásico de Copán mantuvo una organización política y económica centralizada e integrada basada en más que las líneas de parentesco, ideología y ritual. 


\section{BIBLIOGRAFÍA}

- Adams, R. E., \& Jones, R. C. ( 1981 ). Spatial Patterns and Regional Growth among Classic Maya Cities. American Antiquity, 46:301-322.

- Aoyama, K. (1989). Estudio Experimental de las Huellas de Uso sobre Material Lítico de Obsidiana y Sílex. Mesoamérica, 17:185 214.

- Aoyama, K. (1994). Socioeconomic Implications of Chipped Stone from the La Entrada Region, Western Honduras. Journal of Field Archaeology, 21:133 145.

- Aoyama, K. (1995). Microwear Analysis in the Southeast Maya Lowlands: Two Case Studies at Copán, Honduras. Latin American Antiquity, 6:129 144.

- Aoyama, K. (1996). Exchange, Craft Specialization, and Ancient Maya State Formation: A Study of Chipped Stone Artifacts from the Southeast Maya Lowlands. Ph.D. dissertation. University of Pittsburgh.

- Aoyama, K. (1999). Ancient Maya State, Urbanism, Exchange, and Craft Specialization: Chipped Stone Evidence of the Copán Valley and the La Entrada Region, Honduras. Memoirs in Latin American Archaeology No. 12.

- Aoyama, K. (2001). Classic Maya State, Urbanism, and Exchange: Chipped Stone Evidence of the Copán Valley and Its Hinterland . American Anthropologist , 103:346-360.

- Aoyama, K. (2001). Ritos de Plebeyos Mayas en la Cueva Gordon no. 3 de Copán (Honduras) durante el Período Clásico: Análisis de las Microhuellas de Uso sobre la Lítica Menor de Obsidiana. Mayab, 14:5-16.

- Aoyama, K. (2005 ). Classic Maya Warfare and Weapons: Spear, Dart and Arrow Points of Aguateca and Copán. Ancient Mesoamerica, 16:291-304.

- Aoyama, K. (2005). Classic Maya lithic production at Copán, Honduras. Mexicon, 27(2/3):30-37.

- Aoyama, K. (2007). Elite Artists and Craft Producers in Classic Maya Society: Lithic Evidence from Aguateca, Guatemala. Latin American Antiquity, 18:3-26.

- Aoyama, K., Tashiro, T., \& Glascock, M. D. (1999). A Pre-Columbian Obsidian Source in San Luis, Honduras: Implications for the Relationship between Late 
Classic Maya Political Boundaries and the Boundaries of Obsidian Exchange Networks. Ancient Mesoamerca, 10:237-249.

- Ball, J. W., \& Taschek, J. T. (1991). Late Classic Lowland Maya Political Organization and Central Place Analysis: New Insights from the Upper Belize Valley. Ancient Mesoamerica, 2:149 165.

- Bill, C. (1997 ). Patterns of Variation and Change in Dynastic Period Ceramics and Ceramic Production at Copan, Honduras. Ph.D. dissertation. New Orleans: Tulane University.

- Braswell, G. E. (1995). Los artefactos de Obsidiana de Chichén Itzá. Tercer Congreso Internacional de Mayistas. Quintana Roo, México.

- Braswell, G. E., Aoyama, K., McKillop, H. I., \& Glascock, M. D. (2000). Determining the Geological Provenance of Obsidian Artifacts from the Maya Region: A Test of the Efficacy of Visual Sourcing. Latin American Antiquity, $11: 269$.

- Braswell, G. E., Glascock, M. D., \& Neff, H. (1996). The Obsidian Artifacts of Group 10L 2, Copán: Production, Exchange, and Chronology. 61st Annual Meeting of Society for American Archaeology. New Orleans.

- Braswell, G. E., V, E. W., \& Glascock, M. D. (1994). The Obsidian Artifacts of Quelepa, El Salvador. Ancient Mesoamerica , 5:173 192.

- Carrasco, D., Jones, L., \& Sessions, S. (Eds.). (2000). "The Arrival of Strangers": Teotihuacan and Tollan in Classic Maya History. Mesoamerica's Classic Heritage From Teotihuacan to the Aztecs, 465-513.

- Chase, A. F., \& Chase, D. Z. (1996). More Than Kin and King: Centralized Political Organization among the Late Classic Maya. Current Anthropology, 37:803-810.

- Clark, J. E. (1987). Politics, Prismatic Blades, and Mesoamerican Civilization. In J. K. Johnson, \& C. A. Morrow (Eds.), The Organization of Core Technology (pp. 259-284). Boulder: Westview Press.

- Culbert, T. P. (1991). Polities in the Northern Peten, Guatemala . In T. P. Culbert (Ed.), Classic Maya Political History: Hieroglyphic and Archaeological Evidence (pp. 128-146). Cambridge: Cambridge University Press. 
- Demarest, A. A. (1992 ). Ideology in Ancient Maya Cultural Evolution. In A. A. Demarest, \& G. W. Conrad (Eds.), Ideology and Pre Columbian Civilizations (p. 135 157). Santa Fe: School of American Research Press.

- Drennan, R. D. (1991 ). Pre-Hispanic Chiefdom Trajectories in Mesoamerica, Central America, and Northern South America. In T. Earle (Ed.), Chiefdoms: Power, Economy, and Ideology (pp. 263-287). Cambridge: Cambridge University Press.

- Fash, W. L. (1991). Scribes, Warrior and Kings. London: Thames and Hudson.

- Folan, W. J. (1992 ). Calakmul, Campeche: A Centralized Urban Administrative Center in the Northern Peten. . World Archaeology , 24:158-168.

- Fox, J. W., \& Cook, G. W. (1996). Constructing Maya Communities: Ethnography for Archaeology. Current Anthropology, 37:811-821.

- Inomata, T., \& Aoyama, K. (1996 ). Central Place Analyses in the La Entrada Region, Honduras: Implications for Understanding the Classic Maya Political and Economic Systems. Latin American Antiquity, 7:291 312.

- Keeley, L. H. (1980). Experimental Determination of Stone Tool Uses: A Microwear Analysis.

- Mallory, J. K. (1984). Late Classic Maya Economic Specialization: Evidence from the Copán Obsidian Assemblage. Ph.D. dissertation. The Pennsylvania State University.

- Manahan, T. K. (2004). The Way Things Fall Apart: Social Organization and the Classic Maya Collapse of Copan. Ancient Mesoamerica, 15:107-125.

- Marcus, J. (1983). Lowland Maya Archaeology at the Crossroads. American Antiquity, 48:454-488.

- McKillop, H. I. (1989). Coastal Maya Trade: Obsidian Densities at Wild Cane Cay. (P. A. McAnany, \& B. L. Isaac, Eds.) Prehistoric Maya Economies of Belize, 17-56.

- Moholy Nagy, H., Asaro, F., \& Stross, F. H. (1984). Tikal Obsidian: Sources and Typology. American Antiquity, 49:104 117. 
- Sanders, W. T., \& Webster, D. L. (1988). The Mesoamerican Urban Tradition. American Anthropologist, 90:521 546.

- Sharer, R. J. (1983). Interdisciplinary Approaches to the Study of Mesoamerican Highland Lowland Interaction: A Summary View. In A. G. Miller (Ed.), Highland Lowland Interaction in Mesoamerica: Interdisciplinary Approaches (p. 241 263). Dumbarton Oaks Research Library and Collection.

- Sharer, R. J., Schortman, E. M., \& Urban, P. A. (1983). Guatemalan Obsidian: A Preliminary Study of Sources and Quiriguá Artifacts. Quirigua Report II. The University Museum , Philadelphia.

- Sharer, R. J., Traxler, L. P., Sedat, D. W., \& Bell, E. E. (1999). Early Classic Architecture Beneath the Copán Acropolis: A Research Update. Ancient Mesoamerica, 10:3-23.

- Sheets, P. D. (1978). Artifacts In The Prehistory of Chalchuapa, El Salvador. (R. Sharer, Ed.) 2, 1-131.

- Stuart, D. (1992). Hieroglyphs and Archaeology at Copán. Ancient Mesoamerica, 3:169-184.

- Stuart, D. (1995). Alabaster Vessel, El Abra, Honduras. 